Compte rendu de thèse

Sébastien Bédé, Le cluster, réseau territorialisé d'organisations, pour une destination attractive : le cas du tourisme de réunions et de congrès de la région Provence-Alpes-Côte d'Azur

Thèse de doctorat en sciences de gestion de l'Université Nice Sophia Antipolis, dirigée par Jacques Spindler (soutenue le 15 novembre 2013)

\title{
Sébastien Bédé
}

\section{(2) OpenEdition}

\section{Journals}

Édition électronique

URL : http://journals.openedition.org/tourisme/152

DOI : 10.4000/tourisme.152

ISSN : 2492-7503

Éditeur

Éditions touristiques européennes

Édition imprimée

Date de publication : 1 juin 2014

Pagination : 97-102

ISSN : 2109-5671

\section{Référence électronique}

Sébastien Bédé, « Sébastien Bédé, Le cluster, réseau territorialisé d'organisations, pour une destination attractive : le cas du tourisme de réunions et de congrès de la région Provence-Alpes-Côte d'Azur », Mondes du Tourisme [En ligne], 9 | 2014, mis en ligne le 30 septembre 2015, consulté le 22 septembre 2020.

URL : http://journals.openedition.org/tourisme/152 ; DOI : https://doi.org/10.4000/tourisme.152

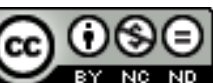

Mondes du tourisme est mis à disposition selon les termes de la licence Creative Commons Attribution - Pas d'Utilisation Commerciale - Pas de Modification 4.0 International. 


\title{
Le cluster, réseau territorialisé d’organisations, pour une destination attractive : le cas du tourisme de réunions et de congrès de la région Provence-Alpes-Côte d'Azur
}

\author{
Thèse de doctorat en sciences de gestion de l'Université Nice Sophia Antipolis, \\ dirigée par Jacques Spindler \\ (soutenue le 15 novembre 20/3)
}

Sébastien BédÉ

[sebastien.bede@em-strasbourg.eu]

D)

armi les études menées sur les relations interorganisationnelles, la structuration du réseau est souvent traitée comme un sujet à part entière (Jamal et Getz, 1995 ; Palmer et Bejou, 1995). De nombreuses approches théoriques ont été développées et appliquées pour analyser ces relations interorganisationnelles : la théorie de la dépendance des ressources (Pfeffer et Salancik, 1978), la théorie des coûts de transaction (Williamson, 1975), les ressources stratégiques (Prahalad et Hamel, 1990), la théorie des réseaux (Granovetter, 1985 ; Gulati, 1998). Les travaux mobilisant ces approches dans le cadre de l'industrie du tourisme soulignent l'im- portance des relations entre les touristes, les entreprises de services et les organisations de gestion de la destination (Tinsley et Lynch, 200I ; Lazzeretti et Petrillo, 2006), mais peu de travaux s'emparent de l'approche par les réseaux pour s'intéresser à la destination touristique.

La gestion d'une destination est considérée dans nos travaux sous l'angle d'une approche stratégique et opérationnelle pour renforcer l'attractivité de territoires dépendant des retombées économiques liées au tourisme. La gestion est fonction de la coordination des parties prenantes de la destination, dont les échanges peuvent concerner de simples informations jusqu'à des collaborations techniques ou commerciales (Baggio, 2007). Pour analyser les relations entre les acteurs d'une destination, notre recherche mobilise les concepts de relations interorganisationnelles et de réseau territorialisé d'organisations de tourisme de réunions et de congrès (TRC) (Christofle, 20 I3), et plus particulièrement celui de cluster.

Les relations interorganisationnelles structurent le réseau et améliorent ses performances en créant des avantages compétitifs (Tremblay, 1998). Cet ensemble de nœuds et de relations structure les réseaux d'entreprises. Porter (1999) définit le cluster comme "un groupe géographiquement proche d'entreprises 
liées entre elles et d'institutions associées relevant d'un domaine donné, entre lesquelles existent des éléments communs et des complémentarités. Son étendue géographique varie d'une seule ville ou d'une région à un pays entier, voire à un réseau de pays voisins [...]." Pour Wolfe et Gertler (2004), il correspond à un réseau asymétrique d'entreprises spécialisées (dominé par une grande entreprise) et ancrées localement (étendue géographique limitée). Toutefois, le succès mondial d'un cluster tel que la Silicon Valley repose en premier lieu sur des relations interorganisationnelles; la proximité géographique y joue un rôle secondaire (Suire et Vicente, 2008 ; Retour, 2008). Certains auteurs tels que Granovetter (2000) et Iammarino et McCann (2006) ont déjà croisé la théorie des réseaux sociaux et l'approche par les clusters. Cependant, pour Hall (2005), la mise en application de cette approche soulève quelques problèmes concernant la mesure de la densité des relations d'un cluster, la description de la chaîne de valeur du tourisme et le rôle du territoire dans la mise en place du cluster.

\section{Problématique}

Bien que les recherches sur les destinations utilisant l'approche par les réseaux sociaux soient nombreuses, peu d'entre elles tentent de mettre en évidence les mécanismes activant la mise en relation des acteurs pour en développer l'attractivité. Selon Clergeau et Violier
(20/3), l'approche par les proximités offre une analyse riche des coopérations et de la mise en réseau des acteurs, cependant "[elle] est très faiblement mobilisée par les chercheurs pour comprendre les dynamiques des clusters touristiques". Ainsi, notre question de recherche est la suivante : dans quelle mesure les différents types de proximité jouent-ils un rôle dans la structuration d'un cluster de tourisme?

Nous questionnons le concept de coordination des acteurs au sein d'un réseau territorialisé d'organisations à travers les différentes formes de proximité (organisationnelle, sociale, cognitive, institutionnelle et géographique) développées par Bouba-Olga et Grossetti (2008). Les différentes formes de proximité trouvent un intérêt particulier en sciences de gestion. D'après Gomez, Rousseau et Vandangeon-Derumez (201 I), "la concentration physique d'individus (dans une organisation) ou d'organisations (sur un territoire) crée des effets de proximité qui ne peuvent être ignorés". De nombreuses études comportent une même problématique, à savoir : "[...] la plus ou moins grande proximité entre les acteurs a des conséquences sur les processus et les performances des organisations" (Gomez, Rousseau et VandangeonDerumez, 20II). Selon Marcelpoil et François (2008), "l'articulation des proximités varie dans le temps et leur nature permet de rendre compte de dysfonctionnements dans l'organisation des stations". L'objectif est alors de mettre en évi- dence les freins à la coordination entre acteurs pour la gouvernance des destinations touristiques. En management stratégique, les chercheurs s'intéressent aux avantages concurrentiels liés à la localisation géographique (Porter, 1990) qui favorisent l'innovation et développent l'attractivité. Ainsi, la proximité peut interroger les relations plus ou moins fortes qui lient une organisation à un réseau. Contrastant avec de nombreuses études, et dans le sillon de Wolfe et Gertler (2004), nous traitons de l'existence potentielle du cluster plutôt que d'en supposer l'existence a priori. Le point de départ de notre étude consiste à identifier les coopérations pour la promotion du TRC à travers les relations interorganisationnelles.

\section{MÉTHOdologie}

Nous avons mobilisé la méthode dite mixte qui est envisageable lorsqu' "une combinaison de données quantitatives et qualitatives peut permettre d'enrichir la compréhension du phénomène étudié et la validité interne de la recherche grâce à la diversification et la triangulation des sources de données qu'elle rend possible" (Avenier et Thomas, 20l I). Dans un premier temps, l'analyse structurale nous permet d'appréhender les propriétés de la structure du réseau (Granovetter, 1985 ; Burt, 1995) à partir de concepts tels que la densité, la centralité et la périphéralité (ces concepts sont définis avec les résultats de l'analyse structurale afin d'en faciliter la compré- 
hension). Notre variable relationnelle repose sur la coopération des acteurs, c'est-à-dire le fait qu'ils partagent des ressources, pour la promotion du tourisme de congrès au niveau régional. Nous avons soumis aux répondants une liste des organisations de la région Provence-Alpes-Côte d'Azur (Paca) sous la forme d'un questionnaire dans lequel ils ont indiqué (et parfois complété) les organisations avec lesquelles ils ont des liens. Les données ont été analysées avec le logiciel Ucinet. Dans un second temps, l'étude discursive nous a permis de questionner les mécanismes sousjacents à la gouvernance d'un réseau en appréhendant la stratégie des acteurs. Les 26 entretiens réalisés représentent 37 heures d'enregistrement et 213 pages de retranscription. Ces entretiens ont fait l'objet d'une analyse de leur contenu afin de comprendre l'importance qu'occupe la stratégie des organisations dans la gouvernance du réseau à partir du logiciel NVivo. La codification des données a été établie à partir de catégories qui correspondent au cadre d'analyse de la gouvernance exposé cidessus.

\section{RéSULtaTS}

Les résultats nous permettent d'établir une tendance des acteurs à se regrouper autour de la ville de Nice et de Marseille ( $c f$. figure 1). Ce phénomène de polarisation peut s'expliquer par les différentes dimensions de la proximité géographique,

\section{Figure 1 : La division de la région Provence-Alpes-Côte d’Azur en territolres touristiques}

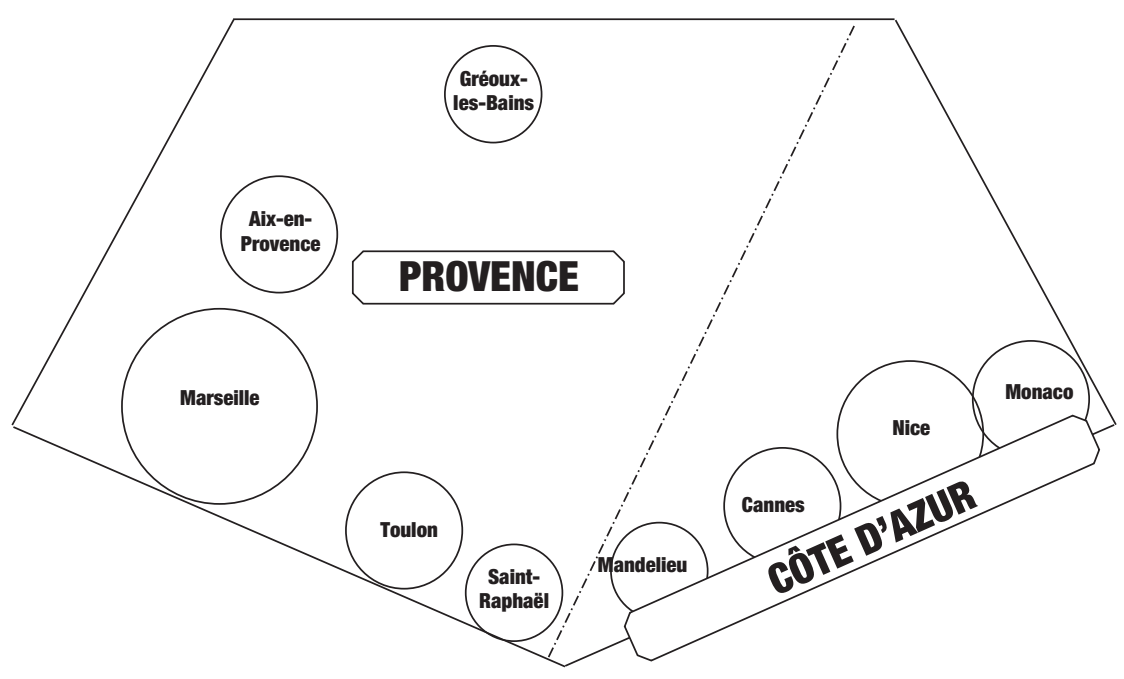

de la proximité de médiation et de celle des ressources immatérielles.

\section{Un phénomène de polarisation}

À Marseille, les acteurs du tourisme de réunions et de congrès ont bénéficié de l'arrivée du TGV. À l'est de la région, ils ont observé les effets du TGV à Marseille et sont conscients des enjeux du rattachement à ce réseau. Le TGV fait aujourd'hui cruellement défaut à la Côte d'Azur. Par conséquent, pour elle, l'aéroport est vital, car il est le seul moyen d'accès performant aux villes situées à l'est de la région. Marseille est donc un nœud articulant, d'une part, les moyens de transport de façon réticulaire en tant que point de jonction et, d'autre part, les échelles territoriales. Marseille a développé son influence grâce au port puis, aujourd'hui, grâce à l'aéroport et au TGV.

\section{Les acteurs-clés du réseau}

La proximité de médiation a permis de mettre en évidence la position centrale des organisations de gestion de la destination (OGD) dans le réseau et leur rôle dans la structuration du réseau. Les élus des collectivités territoriales sont également des intermédiaires. Toutefois, les observations soulignent un manque de considération de la part des élus pour le TRC et des relations de tension entre ces derniers entravant les dispositifs de coopération.

\section{Les ressources du territoire}

La marque, l'image, la culture et l'histoire sont des dimensions qui font référence au territoire comme construit social. Les acteurs sont attachés à leur territoire par une histoire économique plus ou moins liée au tourisme. L'image de la destination est la dimension principale 
sur laquelle les acteurs structurent leurs coopérations. Il est essentiel de noter, d'une part, que l'image et la marque sont diffusées par l'intermédiaire des OGD, et, d'autre part, que les OGD sont perçues comme la dimension principale de la proximité de médiation. Les chartes, garanties d'un niveau homogène de qualité, sont mises en place par les OGD. À l'image de son appellation, la région Provence-Alpes-Côte d'Azur est divisée en territoires composés de deux métropoles à ses extrémités et de villes exerçant une influence démographique, politique et économique importante. Ces territoires véhiculent des images distinctes indépendamment des frontières administratives. La mise en place d'une stratégie pour la promotion régionale du TRC entre en contradiction avec la réalité du territoire comme construit social.

\section{Le cluster à partir des} complémentarités de proximité

Les résultats de notre étude mettent en évidence le déficit de proximité géographique pour activer la coordination au sein de la région Paca. La proximité géographique seule n'assure pas la coordination quand elle n'est pas activée par une proximité organisée. Il est alors nécessaire de s'interroger sur la possibilité qu'un réseau soit construit sur la seule proximité organisée et de s'intéresser à l'importance du rôle de l'histoire et du temps pour construire le réseau local. Toutefois, les résultats de notre étude rendent également manifeste le manque de proximité organisée pour activer la coordination. Par conséquent, la destination touristique au niveau régional ne représente aucune réalité et correspond à des activités dispersées (Torre, 2006). Il n'est pas exclu que la région devienne un cluster et l'initiative du Prides ${ }^{(1)}$ peut orienter la trajectoire du réseau.

\section{Discussion}

Les clusters issus d'initiative publique connaissent des conflits d'intérêts liés aux subventions de la structure de gouvernance du cluster. En effet, la structuration du Prides est fondée sur une logique "top-down", alors que les réseaux locaux sont soutenus par une logique "bottom-up". L'initiative du Prides combine un déplacement du centre de gravité de la proximité de médiation et une déconstruction de la proximité géographique. Ces modifications bouleversent l'organisation régionale du tourisme et contraignent les acteurs à réorganiser la structure du réseau pour s'adapter au changement d'échelle spatiale, à de nouveaux acteurs et à de nouveaux enjeux. La conciliation des actions de chaque membre du réseau dépend alors de leur capacité à s'aligner sur des objectifs communs.

L'organisation du tourisme, définie par les lois ${ }^{(2)}$ promulguées dans le cadre de la décentralisation des compétences en matière de tourisme, prévoit que les acteurs inscrivent leurs actions dans un ensemble cohé- rent en prenant en compte les actions des échelons administratifs adjacents. Dans le cadre de la décentralisation et dans un contexte de réduction des subventions attribuées au comité régional du tourisme Côte d'Azur, la coopération des acteurs locaux avec le Prides fait craindre une perte de pouvoir des collectivités territoriales et des élus locaux. S'il s'avère que les bureaux des congrès locaux sont en doublon ou n'ont plus de légitimité, il sera certainement envisagé de les supprimer. Au regard des enjeux économiques et de l'image du territoire liés au TRC, cette politique régionale génère des conflits qui nuisent aux coopérations entre "techniciens".

Les tensions liées à la conservation de la compétence tourisme font écho à la remise en cause de l'article 72 de la Constitution ${ }^{(3)}$ qui prévoit qu' "aucune collectivité territoriale ne peut exercer une tutelle sur une autre". Ainsi, chaque collectivité dispose de compétences en tourisme et n'entend pas s'en dessaisir. L'article 72 prévoit la désignation d'un "chef de file". Ce point a plusieurs fois été remis en cause. Le 15 mai 2013, la commission des lois a proposé un projet de loi de modernisation de l'action publique territoriale et d'affirmation des métropoles qui propose de désigner "chaque niveau de collectivités territoriales comme chef de file pour la mise en œuvre de compétences nécessitant l'intervention de plusieurs collectivités territoriales d'échelons différents $^{(4)}$ '. Dans ce cadre, il est aujourd'hui prévu que le schéma 


\section{ACTUALITÉ DE LA RECHERCHE}

d'organisation des compétences et de mutualisation des services soit défini conjointement entre le conseil régional et les conseils généraux des départements et que la région assure le rôle de chef de file. Néanmoins, les ambiguités du projet de loi et les multiples modifications apportées depuis 2004 laissent penser que le choix du chef de file dépendra d'un jeu de compromis entre les collectivités territoriales autour de conflits d'intérêts politiques.

L'enjeu du débat qui se joue actuellement afin de définir une collectivité territoriale comme chef de file du tourisme ne peut pas trouver d'issue favorable pour l'ensemble des territoires touristiques. Le découpage territorial est un facteur décisif dans les choix stratégiques de coopération. Toutefois, le changement d'échelle qui serait opéré en impo- sant une référence territoriale calquée sur les limites administratives régionales ou départementales a toutes les chances de ne pas correspondre à chacune des destinations. Parfois, la destination pertinente est dépendante de deux régions ou de deux départements. Dans ce cas, quelle est la solution prévue par l'avant-projet de loi du 10 avril 2013 portant décentralisation et réforme de l'action publique porté par le gouvernement? Aucune.

L'avant-projet de loi portant décentralisation et réforme de l'action publique porté par le gouvernement revêt un enjeu important pour l'attractivité de nos destinations. Il est donc souhaitable que le cluster constitue une solution alternative qui permette de désigner un chef de file. À cette fin, il est important de redistribuer les compétences en matière de tourisme à partir d'une lecture claire de l'activité économique, des relations interorganisationnelles, de l'histoire du territoire, de l'image de la destination, etc.

\section{Notes}

(I) Le Pôle régional d'innovation et de développement économique et solidaire (Prides) a été créé à Marseille en 2008 par la région afin de structurer le réseau d'acteurs du TRC et de faire face à la concurrence.

(2) Les lois du 3 janvier 1987, du 13 juillet 1992, du 27 février 2002 et du 13 août

2004 fixent la répartition des compétences entre l'État, les régions, les départements et les communes.

(3) Article modifié par la loi constitutionnelle n²003-276 du 28 mars 2003 -art. 5.

(4) [http://mww.senat.fr/rap/l I2-580/II25809.html].

\section{Références bibliographiques}

\begin{abstract}
Marie-José AvenIer et Catherine THomas, "Mixer quali et quanti pour quoi faire ? Méthodologie sans épistémologie n'est que ruine de réflexion", Cahiers de recherche du Cerag, juin 2011.

Rodolfo BAGGIO, "What network analysis can reveal about tourism destinations", communication au colloque Complex Networks: from Biology to Information Technology, Pula (Italie), 2-6 juillet 2007.
\end{abstract}

Olivier Bouba-Olga et Michel Grossettl, "Socio-économie de proximité", Revue d'économie régionale \& urbaine, n 3, 2008.
Ronald S. BURT, "Le capital social, les trous structuraux et l'entrepreneur", Revue française de sociologie, vol. 36, 1995.

Cécile Clergeau et Philippe Violier, "Les clusters tourisme : quels enjeux pour les systèmes d'information?", Mondes du tourisme, hors série, mars 2013.

Sylvie CHRISTOFLE, "Tourisme de réunions et de congrès et recherche géographique. Une thématique en devenir", Mondes du tourisme, hors série, mars 2013.

Pierre-Yves GomeZ, Anne RousseAu et Isabelle VANDANGEON-DeRUMEZ, "Distance et proximité. Esquisse d'une problématique pour les organisations", Revue française de gestion, $n^{\circ} 213,2011$. 
Mark Granovetter, "Economic action and social structure: the problem of embeddedness", American Joumal of Sociology, vol. 91, n³, 1985.

Mark Granovetter, "The economic sociology of firms and entrepreneurs", dans Richard Swedberg (dir.), Entrepreneurship: A Social Science View, Oxford University Press, 2000.

Ranjay GULATI, "Alliances and networks", Strategic

Management Journal, vol. 19, n 4, 1998.

Colin Michael HALL, "Rural wine and food tourism cluster and network development", dans Derek R. Hall, Irene Kirkpatrick et Morag Mitchell (dir.), Rural Tourism and Sustainable Business, Channel View Publications, 2005.

Simone IAMMARINO et Philippe MCCANN, "The structure and evolution of industrial clusters: transactions, technology and knowledge spillovers", Research Policy, Elsevier, vol. 3, nº 7 , 2006.

Tazim B. JAMAL et Donald GetZ, "Collaboration theory and community tourism planning", Annals of Tourism Research, vol. $22, n^{\circ} 1,1995$.

Luciana Lazzeretti et Clara S. Petrillo, Tourism Local Systems and Networking, Elsevier, 2006.

Emmanuelle MarcelpoIl et Hugues Françols, "Les processus d'articulation des proximités dans les territoires touristiques. L'exemple des stations de montagne", Revue d'économie régionale \& urbaine, $n^{\circ} 2,2008$.

Adrian Palmer et David Bejou, "Tourism destination marketing alliances", Annals of Tourism Research, vol. 22, n³, 1995. Jeffrey Pfeffer et Gerald R. SALANCIK, The Extemal Control of Organizations: A Resource Dependence Perspective, Harper and Row Publishers, 1978.

Michael E. PORTER, The Competitive Advantage of Nations,

Macmillan, 1990

Michael E. PORTER, La Concurrence selon Porter, Village Mondial, 1999.

C. K. Prahalad et Gary Hamel, "The core competence of the corporation", Harvard Business Review, mai-juin 1990.
Didier Retour, "Pôles de compétitivité, propos d'étape",

Revue française de gestion, $n^{\circ}$ 190, 2008.

Raphaël SUIRE et Jérôme VICENTE, "Théorie économique des clusters et management des réseaux d'entreprises innovantes", Revue française de gestion, $n^{\circ}$ 184, 2008.

Ross TINSLEY et Paul LYNCH, "Small tourism business networks and destination development", International Joumal of Hospitality Management, vol. 20, $n^{\circ} 4,2001$.

André TORRE, "Clusters et systèmes locaux d'innovation. Un retour critique sur les hypothèses naturalistes de la transmission des connaissances à l'aide des catégories de l'économie de la proximité", Régions et Développement, n²4, 2006.

Pascal TREMBLAY, "The economic organization of tourism", Annals of Tourism Research, vol. 25, $n^{\circ}$ 4, 1998.

Oliver E. WILLIAMSON, Markets and Hierarchy. Analysis and Antitrust Implications, Free Press, 1975.

David A. Wolfe et Meric S. Gertler, "Clusters from the inside and out: local dynamics and global linkages", Urban Studies, vol. 4I, nos 5-6, 2004. 